\title{
Training in prison psychiatry
}

\author{
Eluned Dorkins
}

\begin{abstract}
Training in psychiatric work in prisons may not always receive adequate attention, as highlighted in the Reed Report (Department of Health and Home Office, 1992). As a first step in addressing this, a one-day induction procedure has been added to the Induction Programme for the Forensic Senior Registrars at the Regional Secure Unit (RSU) in Bristol. The contents of the programme were determined by discussion among prison medical staff, the Training Department and Forensic Senior Registrars, and are outlined in the following timetable:
\end{abstract}

8.15 Meet at gate

8.30 Accompany prison medical officer on sick parade

9.00 "E" wing adjudication; visit segregation unit

11.00 Meeting with Chaplain

Meeting with "listener" and discussion of scheme

11.30 Meeting with seconded Probation Officer

12.00 Tour of Healthcare Centre

12.30 Lunch

13.30 Security talk

15.00 Healthcare Centre to accompany duty doctor on reception

Three main goals were defined:

(a) To obtain a view of the roles/responsibilities of the prison medical staff and visiting psychiatric staff and where they differ.

(b) To understand the context of practice - i.e. basic operational issues as well as exposure to the prison culture and its attendant problems.

(c) Increase awareness of ethical/legal issues that arise - for example confidentiality; treatment under Common Law; adjudications; food refusal and access to medical records.

Trainees found the day useful but it highlighted some of the underlying tensions and problems of working in such an environment. The lack of independence of visiting medical staff is, for example, indicated by reliance on the prison staff for mobility. The difficulties of sharing information become apparent when the limits of confidentiality are considered. The multidisciplinary team in prison is not synonymous with the multidisciplinary team in the RSU or general psychiatric ward. Should listener schemes have psychiatric input? What if such schemes get abused by inmates?

These issues raise the question as to how trainees can best be prepared for working in the prison environment where in their healthcare role they may come into conflict with the needs of the institution.

\section{Acknowledgement}

I would like to thank the staff at HMP Bristol for their help with this project.

\section{Reference}

DEPARTMENT OF HEALTH AND HOME OFFICE (1992) Review of Health \& Social Services for Mentally Disordered Offenders and Others Requiring Similar Services. Chairman Dr J. Reed. Final Summary Report CM 2088. London: HMSO.

Eluned Dorkins, Senior Registrar in Forensic Psychiatry. The Butler Clinic, Langdon Hospital, Exeter Road, Dawlish, Devon EX7 ONR 